\title{
Novel cognitive ageing rehabilitation with transcranial direct current stimulation
}

\begin{abstract}
Impairment of cognitive function and mobility are commonly found in ageing. The transcranial Direct Current Stimulation (tDCS), has increasingly purposive treatments for cognitive rehabilitation. It is inexpensive, easy to administer after well trained, portable, and home used design considered as the most cost-effective and good compliance therapy. The local effect of calcium-dependent synaptic plasticity of glutamatergic neurons, locally reduce gamma-aminobutyric acid (GABA) neurotransmission, remote effect of the interference with functional connectivity, synchronization, and oscillatory activities in various cortical and subcortical networks, involve in transmembrane ion conductance, membrane structure, cytoskeleton, or axonal transport, degradation of the beta-amyloid and other pathological proteins. In addition, tDCS can enhance axonal regeneration and neurite outgrowth and therefore hypothetically improve functional neuron recovery, produce brain-derived neurotrophic factor (BDNF) that increases synaptogenesis and neurogenesis in the long term, increases glucose metabolism and also be useful to limit the vicious circle of auto-destructive events due to the increased $\mathrm{Ca} 2+$ influx resulting from excessive membrane depolarization or intra-axonal $\mathrm{Na}+$ overload in the context of ischemia or energetic resource failure. The review of several studies of tDCS for cognitive rehabilitation in normal elderly with mild cognitive impairment (MCI) and Alzheimer's disease (AD) are included. More evidences are upcoming in cognitive rehabilitation with tDCS. In conclusion, tDCS is cost-effective and safety for cognitive ageing rehabilitation.
\end{abstract}

Keywords: transcranial direct current stimulation (tDCS), ageing, cognitive rehabilitation
Volume 3 Issue 6 - 2018

\author{
Areerat Suputtitada \\ Physiatrist, Department of Rehabilitation Medicine, Thailand
}

Correspondence: Areerat Suputtitada, Professor, Physiatrist, Department of Rehabilitation Medicine, Faculty of Medicine, Chulalongkorn University \&King Chulalongkorn Memorial Hospital, Bangkok,Thailand, Email prof.areeret@gmail.com, sareratIII |@gmail.com, arerat.su@chula.ac.th

Received: October 29, 2018 | Published: November 15,2018
Impairment of cognitive function and mobility are commonly found in ageing. Walking is cognitive-motor task essential to daily activities. Dual tasking is the ability to do two tasks in the same time. It is one of the executive resources within working memory which is the prediction of cognitive decline and fall risk. The impairment of dual tasking ability is commonly found as the early symptom of Alzheimer's disease (AD). Around 5\% to $10 \%$ of ageing with mild cognitive impairment (MCI) develop dementia each year which incidence is expected to double by the year 2050. "Cognitive aging" is the ageing process of cognitive decline. It is unique to each individual, since the differences in genetic, life experiences, and educational level. The declining of memory start from episodic memory during mid-life, then semantic memory and executive function decrease when elder Additionally, slow psychomotor processing and difficulty in focus attention and/or multitasking. There are evidences of neuroimaging for cognitive-motor tasks activating the cortical networks including the dorsolateral prefrontal cortex (DLPFC) for executive function. The tDCS with anodal electrode at the DLPFC may improve cognitive function and mobility in older adults. ${ }^{1-4}$

Cognitive impairments from brain atrophy means cortical thinning or decreased gray matter volume. The most common areas of atrophy are prefrontal cortex, temporal lobes, and hippocampus. The frontal lobe, which is lastly mature at the age of 25 years old and thin firstly with age. It has an important role in higher-order cognitive processes and a variety of critical executive functions as planning, decision making, problem-solving, and working memory. The process of increasing free radicals and dendritic spinal loss resulted from demyelination or lesions to white matter tracts, imbalances in dopamine and serotonin, decreasing of the brain-derived neurotrophic factor, increasing of monoamine oxidase, and deposition of amyloid beta $(\mathrm{Ab}) .^{5,6}$

Transcranial Direct Current Stimulation (tDCS) has increasingly purposive treatments for neurorehabilitation including pain and ageing rehabilitation, compared to other noninvasive neuromodulations. It is inexpensive, easy to administer after well trained, portable, considered as the most cost-effective and good compliance therapy. It can either enhance or suppress cortical excitability by a weak and constant direct current applied to the brain according to the montage of 10-20 system. It has effect for several hours after the stimulation, depending on several factors. The protocols of stimulation are varied in each indication and usually need to do at least 10 days for long time effects.

\section{How tDCS works on cognitive function}

The effect of tDCS on neurons are as the followings; (1) A subthreshold shift of resting membrane potentials towards depolarization or hyperpolarization at the synaptic level, depending on current flow direction relative to axonal orientation. (2) Reduce gamma-aminobutyric acid (GABA) neurotransmission, regardless of stimulation polarity and impact on glutamatergic plasticity. (3) The interference with functional connectivity, synchronization, and oscillatory activities in various cortical and subcortical networks. (4) The modulation of resting membrane potential more generally along the whole axons resulting in non-synaptic effects which made long- 
lasting after-effects. (5) The changes of conformation and function of various axonal molecules, involved in transmembrane ion conductance, membrane structure, cytoskeleton, or axonal transport. (6) the changes in non-neuronal tissues in the brain, including endothelial cells, lymphocytes, or glial cells, influencing on the inflammatory response. (7) The beta-amyloid and other pathological proteins can be modified to slower the process of AD. (8) The enhancement of axonal regeneration and neurite outgrowth will improve functional recovery. (9) The production of brain-derived neurotrophic factor (BDNF) that increases synaptogenesis and neurogenesis in long term effects. (10) The increasing of cerebral glucose metabolism induced by increasing neuronal activity. (11) Limit the vicious circle of autodestructive events due to the increased $\mathrm{Ca} 2+$ influx resulting from excessive membrane depolarization or intra-axonal $\mathrm{Na}+$ overload in the context of ischemia or energetic resource failure. ${ }^{6-22}$

\section{Evidences of tDCS for cognitive aging rehabilitation}

tDCS is safe to use and has shown potential for enhancing cognition in several studies as the followings; In 2008, Ferrucci et al studied the cognitive effect of tDCS over the temporoparietal areas in 10 patients with $A D$ with anodal tDCS, cathodal tDCS and sham tDCS in 3 sessions for 30 minutes. This study revealed that anodal tDCS over the temporoparietal areas can specifically affect the recognition memory performance in $\mathrm{AD}^{23}$

In 2012, Boggio et al. ${ }^{24}$ studied cognitive functions in $15 \mathrm{AD}$ patients. $2 \mathrm{~mA}$ tDCS for 30 minutes, 5 consecutive days. The tDCS current was delivered bilaterally through two scalp anodal electrodes placed over the temporal regions and a reference electrode over the right deltoid muscle. This study revealed that bilateral anodal tDCS over the bilateral temporal regions improve visual recognition memory for at least 4 weeks after therapy. ${ }^{24}$

In 2013, Park et al randomized 40 elderly to receive sham or active tDCS during computer-assisted cognitive training 10 sessions (5 days/per week for 2 weeks) for $30 \mathrm{~min}$ a day. The active $2 \mathrm{~mA}$ tDCS current and sham tDCS using two stimulators or two anodes were placed over F3 and F4 (bilateral prefrontal cortex) and two cathodes were placed on the non-dominant arm for $30 \mathrm{~min}$ a day. There are significant improvements in digit span forward tests in both groups. The effect on verbal working memory accuracy improvement lasted for 28 days after tDCS. This study revealed that bilateral anodal tDCS over bilateral prefrontal cortex enhance cognitive function in healthy elderly and lasted over the stimulation period. ${ }^{25}$

In 2013, Meinzer et al. ${ }^{26}$ used a task-based fMRI study with tDCS examined the effects of tDCS on language function in 20 healthy elderly (age 60-76) and healthy adults (aged 19-31). Active tDCS with $1 \mathrm{~mA}$ for 17 min followed by a semantic word generation task. The anode was placed over the left inferior frontal gyrus (IFG) (10-20 EEG system corresponding to the intersection of T3-F3 and F7-C3 and the midpoint between F7-F3) and the cathode on the contralateral supraorbital region. During Sham tDCS, there are lower semantic word-generation tasks ability in healthy elderly associated with enhanced task-related activity in bilateral IFG activation. The active tDCS in elderly produced significantly higher performance compared to the young adults, and significantly reduced task-related hyperactivity in the bilateral prefrontal cortex (PFC). Increased connectivity was also observed between the left IFG and the language related cortical areas during the resting state in active tDCS. This study revealed that $\mathrm{tDCS}$ enhance cognitive function in elderly with direct impact on underlying neural response patterns. ${ }^{26}$

In 2014, Hartly et al. ${ }^{27}$ studied the effects of tDCS on error awareness in 96 healthy elderly, divided into 4 groups with 24 healthy elderly per group. The study tested the influence of current polarity and electrode location (anode over F3 or F4, and cathode over $\mathrm{Cz}$ ), on error monitoring. During $1 \mathrm{~mA}$ tDCS application, the elderly performed a computerized test of error awareness ( 5 blocks, each 7.5 min and 1-min resting time within each block), a Go/No-go response inhibition task that required constant monitoring to detect errors. The group with anode stimulation over the right DLPFC (F4) was the only group to experience improved error detection during the task. This study revealed that the right DLPFC tDCS have a larger role on error awareness. ${ }^{27}$

In 2015, Meinzer et al. ${ }^{28}$ performed a double-blind, cross-over, sham-controlled study. Anodal-tDCS $1 \mathrm{~mA}$, for 20 minutes stimulated at the left inferior frontal cortex during task-related and resting-state functional magnetic resonance imaging (fMRI) to assess its impact on cognition and brain functions in MCI. During sham stimulation, patients produced fewer correct semantic-word-retrieval responses than matched healthy controls, which was correlated with hyperactivity in bilateral prefrontal regions. Anodal-tDCS significantly improved performance to the level of controls, reduced task-related prefrontal hyperactivity and resulted in "normalization" of abnormal network configuration during resting-state fMRI. This study revealed that anodal-tDCS exerts beneficial effects on cognition and brain functions in MCI, and repeated stimulation sessions can produce the sustained improvement of cognition. ${ }^{28}$

In 2016, Manenti et al. ${ }^{29}$ studied in 20 patients with PD, 10 patients assigned to anodal $2-\mathrm{mA}$ tDCS for 25 minutes during physical therapy and 10 patients to sham tDCS for 25 minutes during physical therapy. Both groups are done for 2 weeks. The Parkinson's Disease Cognitive Rating Scale and verbal fluency test performances increased only in the anodal tDCS with a stable effect at 3-month follow up. This study revealed that anodal tDCS is a novel therapy for PD patients with mild cognitive impairment. ${ }^{29}$

In 2016, Stephens et al..$^{30}$ randomized 90 healthy older adults to receive sham, $1 \mathrm{~mA}$, or $2 \mathrm{~mA}$ of tDCS stimulation for $15 \mathrm{~min}$ during five sessions of working memory training. The anode electrode was placed over the right DLPFC (F4), and the cathode was placed over the contralateral cheek. All participants showed improvements in the trained verbal and visual working memory tasks. This study revealed that anodal tDCS can increase far transfer benefits (processing speed, cognitive flexibility, arithmetic) at 1 month after anodal tDCS at the right DLPFC (F4)..$^{30}$

In 2016, Yun et al. ${ }^{31}$ studied the effects of repeated tDCS on glucose metabolism and cognitive performance in $16 \mathrm{MCI}$. The $2 \mathrm{~mA}$ anodal tDCS over left DLPFC (F3) and cathodal tDCS over the right DLPFC (F4) with bilateral frontal stimulation were applied for $30 \mathrm{~min}, 3$ times per week for 3 weeks. Using Positron emission tomography (PET), revealed a significant increase in cerebral metabolic activity in the medial prefrontal cortex, precuneus, midtemporal regions, and the anterior cingulate cortices in the anodal tDCS group. Multifactorial Memory Questionnaire (MMQ) was performed to assess the participant's subjective memory functioning. MMQ scores and glucose metabolism were significantly improved only in the anodal tDCS group. This study revealed that active tDCS can change the 
regional brain metabolism as well as transient memory function in MCI. $^{31}$

\section{Safety of tDCS}

There is no report evidences of brain damage, seizure or syncope when treatment with tDCS. The only side effects are local skin and scalp effects as irritation, itching, redness.

\section{Conclusion}

tDCS is cost-effective and safety for cognitive ageing rehabilitation. The evidences of tDCS appeared less than 20 years, it is emerging novel non- invasive brain stimulation in both research and clinical settings. As a safe, painless, inexpensive treatment for modulating the excitability of brain tissue, tDCS has strong potential for application in cognitive ageing rehabilitation. The succeed of tDCS treatment depend on the knowledge and experiences of the physicians to select the patients, put the correct area of 10-20 EEG montage and select the protocols.

\section{Acknowledgements}

None.

\section{Conflict of interest}

Author declares that there is no conflict of interest.

\section{References}

1. Seidler RD, Bernard JA, Burutolu TB, et al. Motor control and aging: links to age-related brain structural, functional, and biochemical effects. Neurosci Biobehav Rev. 2010;34(5):721-733.

2. Yogev SG, Hausdorff JM, Giladi N. The role of executive function and attention in gait. Mov Disord. 2008;23(3):329-342.

3. Montero OMM, Sarquis AY, Speechley M, et al. Association of dualtask gait with incident dementia in mild cognitive impairment: results from the gait and brain study. JAMA Neurol. 2017;74(7):857-865.

4. Montero OM, Verghese J, Beauchet $\mathrm{O}$, et al. Gait and cognition: a complementary approach to understanding brain function and the risk of falling. J Am Geriatr Soc. 2012;60(11):2127-2136.

5. Salat DH, Buckner RL, Snyder AZ, et al. Thinning of the cerebral cortex in aging. Cereb Cortex. 2004;14(7):721-730.

6. Gomes OJ, Indahlastari A, Fried PJ, et al. Woods aj.non-invasive brain stimulation: probing intracortical circuits and improving cognition in the aging brain. Front Aging Neurosci. 2018;(8):10:177.

7. Vacas SM, Stella F, Loureiro JC, et al. Non-invasive brain stimulation for behavioural and psychological symptoms of dementia: a systematic review and meta-analysis. Int J Geriatr Psychiatry. 2018.

8. Liu CS, Rau A, Gallagher D, et al. Using transcranial direct current stimulation to treat symptoms in mild cognitive impairment and Alzheimer's disease. Neurodegener Dis Manag. 2017;7(5):317-329.

9. Lawrence BJ, Gasson N, Bucks RS, et al. Cognitive training and noninvasive brain stimulation for cognition in parkinson's disease: a meta-analysis. Neurorehabil Neural Repair. 2017;31(7):597-608.

10. Dinkelbach L, Brambilla M, Manenti R, et al. Non-invasive brain stimulation in Parkinson's disease: exploiting crossroads of cognition and mood. Neurosci Biobehav Rev. 2017;75:407-418.

11. Wiegand A, Nieratschker V, Plewnia C. Genetic modulation of transcranial direct current stimulation effects on cognition. Front Hum
Neurosci. 2016;10:651.

12. Bikson M, Grossman P, Thomas C, et al. Safety of transcranial direct current stimulation: evidence based update 2016. Brain Stimul. 2016;9(5):641-661.

13. Dedoncker J, Brunoni AR, Baeken C, et al. The effect of the intervalbetween-sessions on prefrontal transcranial direct current stimulation (tDCS) on cognitive outcomes: a systematic review and meta-analysis. J Neural Transm (Vienna). 2016;123(10):1159-1172.

14. Elsner B, Kugler J, Pohl M, et al. Transcranial direct current stimulation (tDCS) for improving activities of daily living, and physical and cognitive functioning, in people after stroke [Review]. Cochrane Database Syst Rev. 2016;3:CD009645.

15. Savic B, Meier B. How transcranial direct current stimulation can modulate implicit motor sequence learning and consolidation: a brief review. Front Hu Neurosci. 2016;10:26.

16. Hill AT, Fitzgerald PB, Hoy KE. Effects of anodal transcranial direct current stimulation on working memory: a systematic review and metaanalysis of findings from healthy and neuropsychiatric populations. Brain Stimul. 2016;9(2):197-208.

17. Hsu WY, Ku Y, Zanto TP, et al. Effects of noninvasive brain stimulation on cognitive function in healthy aging and Alzheimer's disease: a systematic review and meta-analysis. Neurobiol Aging. 2015;36(8):2348-2359.

18. Horvath JC, Forte JD, Carter O. Quantitative review finds no evidence of cognitive effects in healthy populations from single-session transcranial direct current stimulation (tDCS). Brain Stimul. 2015;8(3):535-550.

19. Miniussi C, Ruzzoli M. Transcranial stimulation and cognition. Handb Clin Neurol. 2013;116:739-750.

20. Krause B, Márquez RJ, Cohen KR. The effect of transcranial direct current stimulation: a role for cortical excitation/inhibition balance? Front Hum Neurosci. 2013 24;7:602.

21. Miniussi C, Harris JA, Ruzzoli M. Modelling non-invasive brain stimulation in cognitive neuroscience. Neurosci Biobehav Rev. 2013;37(8):1702-1712.

22. Jacobson L, Koslowsky M, Lavidor M. tDCS polarity effects in motor and cognitive domains: a meta-analytical review. Exp Brain Res. 2012;216(1):1-10.

23. Ferrucci R, Mameli F, Guidi I, et al. Transcranial direct current stimulation improves recognition memory in Alzheimer disease. Neurology. 2008;71(7):493-498.

24. Boggio PS, Ferrucci R, Mameli F, et al. Prolonged visual memory enhancement after direct current stimulation in Alzheimer's disease. Brain Stimul. 2012;5(3):223-230.

25. Park SH, Seo JH, Kim YH, et al. Long-term effects of transcranial direct current stimulation combined with computer-assisted cognitive training in healthy older adults. Neuroreport. 2014;25(2):122-126.

26. Meinzer M, Lindenberg R, Antonenko D, et al. Anodal transcranial direct current stimulation temporarily reverses age-associated cognitive decline and functional brain activity changes. $J$ Neurosci. 2013;33(30):12470-12478.

27. Harty S, Robertson IH, Miniussi C, et al. Transcranial direct current stimulation over right dorsolateral prefrontal cortex enhances error awareness in older age. $J$ Neurosci. 2014;34(10):3646-3652.

28. Meinzer M, Lindenberg R, Phan MT, et al. Transcranial direct current stimulation in mild cognitive impairment: behavioral effects and neural mechanisms. Alzheimers Dement. 2015;11(9):1032-1040. 
29. Manenti R, Brambilla M, Benussi A, et al. Mild cognitive impairment in Parkinson's disease is improved by transcranial direct current stimulation combined with physical therapy. Mov Disord. 2016;31(5):715-724.

30. Stephens JA, Berryhill ME. Older adults improve on everyday tasks after working memory training and neurostimulation. Brain Stimul. 2016;9(4):553-559.

31. Yun K, Song IU, Chung YA. Changes in cerebral glucose metabolism after 3 weeks of noninvasive electrical stimulation of mild cognitive impairment patients. Alzheimers Res Ther. 2016;8(1):49. 